\title{
Currículo e Identidad: Entre la Escuela y la Cultura'
}

\author{
Curriculum and Identity: Between School and Cultural
}

\section{Currículo e Identidade: entre a escola e Cultural}

Recibido: mayo de 2013

Aceptado: agosto de 2013
Aldo Iván Parra ${ }^{2}$

Christian Camilo Fuentes Leal ${ }^{3}$

\section{Resumen}

En este taller se pretende reflexionar sobre cómo un modelo de currículo puede genera una determinada identidad de los individuos, el conflicto entre el modelo de identidad presentado por la escuela y el modelo presentado por la cultura del estudiante, para dicha tarea se presentará a los asistentes diferentes situaciones donde se evidencie dicha tensión, se preguntará sus reflexiones y qué tipo de actitudes tomarán con respecto a los elementos encontrados en dichas situaciones y posteriormente se presentarán algunas concepciones del currículo planteados por Bishop $(1999,2005)$ y Stenhouse (2003) las cuales podrían ayudar para la superación de dicha disyunción.

Palabras clave: Currículo; Evaluación del currículo; Etnomatemática; Investigación e innovación en Educación Matemática.

\begin{abstract}
This workshop aims to reflect on how a curriculum model can generate a certain identity of individuals, the conflict between identity model presented by the school and the model presented by the culture of the student, that task will be presented to attendees different situations where there is evidence that stress, reflections and wonder what kind of attitudes taken with respect to the elements found in such situations and then present some concepts of the curriculum presented by Bishop $(1999,2005)$ and Stenhouse (2003) which could help to overcome this disjunction.
\end{abstract}

\section{Keywords:}

Curriculum, curriculum evaluation; Ethnomathematics, Research and Innovation in Mathematics Education.

\section{Resumo}

Este workshop tem como objetivo refletir sobre a forma como um modelo de currículo pode gerar uma certa identidade dos indivíduos, o conflito entre o modelo de identidade apresentado pela escola eo modelo apresentado

1 Articulo de Investigación.

2 Centro Indígena de Investigaciones Interculturales de Tierradentro. Colombia. Contacto: aiparras@unal.edu.co

3 Universidad Distrital Francisco José de Caldas. Bogotá, Colombia. Contacto: ccfuentes@udistrital.edu.co 
pela cultura do aluno, essa tarefa será apresentado aos participantes diferentes situações em que há evidências de que o estresse, reflexões e saber que tipo de atitudes tomadas em relação aos elementos encontrados em tais situações e, em seguida, apresentar alguns conceitos do currículo apresentado pelo Bispo $(1999,2005)$ e Stenhouse (2003), que poderia ajudar a superar essa disjunção.

Palavras-chave: Currículo, avaliação curricular; Etnomatemática, Investigação e Inovação em Educação Matemática.

\section{Introducción}

Cualquier mirada al currículo, encontrará en las intencionalidades de este la construcción idealizada de un sujeto, apto para mantener los valores de la sociedad que define ese currículo. El objetivo es que la persona que se somete a un proceso educativo, al salir de él se desenvuelva idóneamente dentro de una visión del mundo especifica que sustenta esa sociedad, y por ello se establecen una serie de procesos, medios y fines a alcanzar, se definen entonces contenidos, saberes, valores a ser desarrollados en la persona, para que se torne sujeto o ciudadano ${ }^{4}$. Consideramos que los elementos al interior del currículo se establecen con el fin dotar a la persona de una serie de herramientas que le permiten pertenecer a la sociedad; ante lo cual preguntamos, ¿qué es esto sino generar una identidad especifica en la persona?, ¿cómo pretender que esta serie de "contenidos" a ser "transmitidos" no implica una impronta determinante en la racionalidad de la persona, en su forma de discriminar lo que considera importante, pensable y comunicable?, ¿es posible para un ser humano permanecer independiente de la serie de saberes que la sociedad y su institucionalidad le están indicado como indispensables?

En ese orden de ideas no hay mayores discrepancias con reconocer una fuerte relación entre las finalidades de la escuela objetivadas dentro del currículo y el proceso de construcción de identidad del sujeto que se da al interior de cualquier cultura. Sin embargo esta relación está bastante lejos de ser armónica o de subordinación toda vez que la escuela ha ganado una dinámica propia, estableciendo una tradición, todo un andamiaje burocrático y conceptual que ya no puede considerarse subalterno o funcional a los valores de la sociedad, sino a los intereses de las fuerzas que detenten el poder gubernamental.

\section{Marco teórico}

Esta situación ha sido recurrentemente considerada por estudios en Etnomatemática, autores como D’ambrosio (1986, 1994), Knijnik (1998), Jaramillo (2011) presentan la disyunción entre el conocimiento escolar y el extraescolar, donde se llega a extremos de invalidar la escuela como centro de saberes útiles para la vida, mostrándola únicamente como un espacio donde se obtienen certificaciones que permitirán buscar empleo, espacio - donde realmente se aprenderá lo necesario para vivir; en pocas palabras, al configurar un mundo innecesario, irreal, la escuela sólo habla de sí misma y específicamente la asignatura de matemática no es más que una serie de contenidos abstrusos, que no tienen más vida y aplicación que el aula da clase Lins (2004).

Tanto a nivel de contenidos como a nivel de procesos de enseñanza y habilidades a desarrollar, la formación de la escuela cambia asincrónicamente con las demandas de la sociedad, para el caso de la escuela urbana y pública "habitual", podríamos decir que hay una disonancia entre el sujeto ideado por el currículo y la identidad cultural actual en los centros urbanos, basta ver la aparente omnipresencia de los computadores y demás dispositivos electrónicos, disponibles para hacer cálculos tediosos que la escuela persiste en realizar manualmente, destinándoles gran parte del tiempo escolar y entronizandolos como

4 Esto puede ejemplificarse desde el trivium y el quadrivium, hasta las recientes ideas de educación para la democracia y los discursos sobre competencias. 
necesarios, indispensables justificados con frases del estilo: “¡y qué harán si no tienen la maquinita a mano?".

En el mismo sentido podemos observar el manejo que da la escuela a las recientes fuentes de acceso al conocimiento, se han multiplicado los libros de texto con diversos enfoques, aparecen blogs, libros digitales, materiales interactivos, servicios como Wikipedia, Google, Youtube o Wolfram, todo lo anterior brinda información de manera inmediata y muy variada, ¿ el currículo puede seguir pensándose desde el presupuesto de que la escuela (o el libro escogido por ella) es el único lugar donde puede obtenerse la información?. Sin embargo, cuando consideramos escuelas no habituales, ubicadas en lugares periféricos (por ejemplo en comunidades indígenas) la relación entre cultura y currículo por la construcción de identidades llega a ser de antagonismo, valores como la espiritualidad, la organización colectiva, la relación con la naturaleza, tan preciados por las comunidades indígenas vienen a ser amenazados por el currículo, y particularmente con matemática por la implantación de un esquema de racionalidad que funge como el único posible, que crea la necesidad de cuantificar y medir atributos en función de la utilidad económica (time is money) D'Ambrosio (2012), incluso dentro la misma cultura occidental presentando el currículo como un ente artificial por medio de la división del conocimiento en disciplinas.

Algunos autores han presentado algunas alternativas para superar la divergencia entre la identidad y el currículo, Stenhouse (2003) se presenta el concepto de currículo como una forma de trabajo colaborativo, mostrándolo como un instrumento para la transformación de la enseñanza y la acción pedagógica, por medio de la investigación de la misma práctica, para el autor el currículo constituye un cuerpo complejo, pues expresa toda una visión de lo que es el conocimiento y el proceso de educación, un marco donde el profesor puede desarrollar nuevas habilidades, que se pueden relacionar con las concepciones del conocimiento y el aprendizaje.

Para el autor, el currículo no es una mera selección resultados del conocimiento y de la cultura, sino que implica una visión educativa del conocimiento, además es un instrumento de transformación tanto de los profesores como de los estudiantes. Se concibe el currículo como un marco en el que hay que resolver problemas especificos en situaciones puntuales y concretas, el nuevo enfoque de currículo presentado por el autor es construido a partir de una nueva concepción de la profesión del docente y la búsqueda de nuevas metodologías de investigación, éstas ideas están enmarcadas por la relación entre teoría/pensamiento del profesor/acción.

Por otra parte Bishop $(1999,2005)$ hace una crítica al currículo dirigido únicamente al desarrollo de técnicas, e invita a la transformación del currículo en matemáticas, en primer lugar hay que cambiar el rol del profesor a ser un enculturador matemático, él debe pasar de un ente de transformación de ideas matemáticas, a considerar tanto factores sociales y sociológicos presente en el aula, algunos de éstos elementos son, el reconocimiento del papel del profesor como validador y legitimador en el aula de clase, la adopción del modelo antropológico social por medio del acercamiento de los conocimientos extraescolares, estimular a los estudiantes para la articulación de sus conocimientos, a convalidar entre estudiantes, la diferentes simbolizaciones, además de ser consientes sobre los valores de los estudiantes con respecto al conocimiento matemático.

El autor propone la enseñanza por proyectos que relacionen matemáticas, sociedad y cultura, pues este tipo de propuestas genera la exploración de diferentes situaciones sociales y físicas, la comprensión de diferentes modelos matemáticos para la resolución de diferentes situaciones, el estimulo a la adopción de una perspectiva crítica de la sociedad.

Desde esta perspectiva, presenta las matemáticas como una construcción social intrínseca a la vida de los estudiantes, que termina formando la sociedad, donde la educación matemática debería llegar a ser el vehículo para introducir a los estudiantes al conjunto de situaciones que encaran los ciudadanos que quieren vivir en el futuro en una sociedad democrática.

\section{Descripción de las actividades}

Para la elaboración del taller serán presentadas algunas situaciones cotidianas de aulas ${ }^{5}$, que evidencian las relaciones de tensión entre currículo y cultura, 
buscando indagar posicionamientos personales y suscitar reflexiones en los asistentes, para posteriormente entrar en contacto con los diferentes elementos presentados por Bishop $(1999,2005)$ y Stenhouse (2003), como alternativas de trabajo.

\section{Conclusiones}

En la propuesta de Stenhouse (2003) se hace una crítica a las propuestas curriculares basadas en la pre-especificación de los objetivos como punto de partida del desarrollo de un currículo, pues esta característica es poco operativa para el profesor, imposibilitando que las ideas centrales de la propuesta currícular se lleven a la práctica. El autor presenta un modelo curricular por procesos, como alternativa al de los objetivos, pretendiendo relacionar el respeto a la naturaleza del conocimiento y a su metodología, la toma en consideración del proceso de aprendizaje y un enfoque coherente del proceso de enseñanza con relación a los dos elementos anteriormente mencionados, es decir que desde esta perspectiva la mejora de la calidad de la enseñanza ocurrirá por un proceso experimental de la propia práctica de los profesores y no por alguna imposición dogmatica.

Esta iniciativa y la de Bishop, pretenden cuestionar la forma como tradicionalmente es abordado en el aula de clase el conocimiento matemático escolar como único y universal, presentan la escuela como un espacio de diálogo y de debate, lugar donde se da cabida a las subjetividades de los estudiantes, dado que la escuela se configura como un espacio donde la diversidad cultural debe ser atendida, comprendida y asumida, asimismo el abordar la currículo en matemáticas desde una perspectiva sociocultural, implica complejidad, pues existen diferentes tensiones, generadas por la dicotomía a la que nos enfrentamos los investigadores y los maestros, en donde debemos atender, por un lado, a la diversidad cultural de los alumnos, y otro, a los procesos homogeneizadores internos y externos a las instituciones escolares, ésta es resultado del modelo neoliberal en los procesos educativos.

\section{Referencias}

Bishop, A. (2005). Aproximación sociocultural a la educación matemática. Santiago de cali: Universidad del valle.

Bishop, A. (1999). Enculturación matemática, la educación matemática desde una perspectiva sociocultural. Barcelona: Paidós.

D’ Ambrósio, U. Da realidade à ação: reflexões sobre a educação matemática. São Paulo: Summus, 1986

D’Ambrosio, U. (1994). A etnomatemática no processo de construcão de uma escola indígena. Em Aberto, 14 (63), 93 - 99.

D'Ambrosio, U. (2012) The Program Ethnomathematics: Theoretical basis and the dynamics of cultural encounters. Cosmopolis. A Journal of Cosmopolitics/Revue de cosmopolitique (Ghent) vol 2012-3-4, pp.13-41

Jaramillo, D. (2011) Dialogía entre prácticas sociales y prácticas escolares en la escuela indígena. Memorias de la XIII Conferencia Interamericana de educacion matemática. Recife, Brasil.

Knijnik, G. (1998).Educação matemática e os problemas da "vida real". In: CHASSOT, Àtico; OLIVEIRA, Renato J. de (Orgs). Ciência, ética e cultura na educação. São Leopoldo: UNISINOS, 1998. p.123-134

Lins, R. (2004) Matemática, monstros, significados e educação matemática. En M.A.V. Bicudo y M.C. Borba (eds.), Educação Matemática: pesquisa em movimento, São Paulo, Cortez, pp. 92-120.

Stenhouse, L. (2003). Investigación y desarrollo del curriculum. Madrid: Morata. 